\title{
LES DOSES DE SUCCINYLCHOLINE EMPLOYÉES EN ANESTHÉSIE SONT-ELLES TROP ÉLEVEES?*
}

\author{
Gaston Comtois, M.D., $\dagger$ Emile Bertho, M.D., F.R.C.S.(C.), $\ddagger$ AND \\ Alexandre TARDlF, M.D. $\S$
}

DANS LE LABORATOIRE de chirurgie expérimentale où la succinylcholine est très employée, spécialement pour prélever du sang chez les chiens, nous sommes impressionnés chaque jour par les doses minimes et efficaces de cette drogue comparées aux doses élevées employées en chirurgie humaine. Nous sommes: également surpris de constater, dans les salles de réveil, la cyanose, parfois légère mais existante, des extrémités chez les opérés, et cette cyanose peut se prolonger une demi-heure et parfois même une heure. Nous sommes convaincus que la succinylcholine, ou les curarișants en sont responsables pour une large part. Il existe évidemment d'autres médicaments à action dépressive sur les centres respiratoires (barbituriques, narcotiques, etc.) mais l'action prolongée ${ }^{1} \mathrm{de}$ la succinylcholine sur les muscles de la respiration aboutit à une ventilation imparfaite; et cette action est d'autant plus prolongée que l'état de l'opéré est détérioré et que des doses répétées de succinylcholine ont été employées.

Il faut même aller plus loin, c'est-à-dire incriminer la succinylcholine de certaines complications pulmonaires, atélectasie, broncho-pneumonie, etc., surtout chez les gens âgés.

Après un bref historique sur la succinylcholine, sur sa constitution chimique, sur son mode d'action ainsi que sur son sort dans l'organisme, seront exposées les méthodes expérimentales employées, ainsi que les résultats obtenus.

\section{Historique}

Dès 1906, Hunt et Traveau ${ }^{2}$ avaient employé la succinylcholine chez les animaux de laboratoire. Ils n'avaient cependant pas parlé de son activité de bloquage neuro-musculaire. Cette propriété de la succinylcholine est demeurée inconnue pendant plus de 40 ans. Ce n'est qu'en 1949 que l'action curarisante de la drogue fut décrite indépendamment par les Italiens, les Anglais et les Américains. Bovet et ses collaborateurs décrivaient en 1949 les propriétés de la succinylcholine et publiaient en $1951^{3}$ leurs recherches sur les poisons curarisants de synthèse. Philipps, publiait également en 1949 un travail sur les substituts synthétiques du curare. ${ }^{4}$ Ces deux chercheurs, tentaient de démontrer l'action

*Travail présenté au congrès des anesthésistes de la Province de Québec tenu à l'Hôtel-Dieu St-Vallier de Chicoutimi le 28 mai 1960 , et effectué gràce à la collaboration des autorités adminis. tratives de l'Hôtel-Dieu St-Vallier de Chicoutimi.

†Chef du Département d'Anesthésie.

†Chef du Service de Chirurgie Cardo-vasculaire (expérimentale et clinique).

$\S R$ Résident dans le Département d'Anesthésie. 
du "bloquage myoneural" de cette drogue. En 1951, Low et Tammelin ${ }^{5}$ mirent au point une nouvelle technique pour préparer la succinylcholine squs forme de poudre cristalline exempte d'impureté.

\section{Chimie et Mode D'ACTION ${ }^{1,6,13}$}

La succinylcholine, qui semble à première veu un "paradoxe pharmacologique," a une structure chimique correspondant à une double acétylcholine.

\section{Formule}

Succinylcholine:

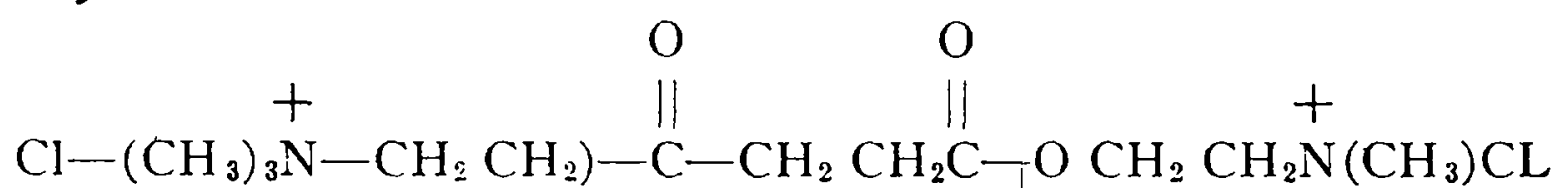

Acetylcholine:

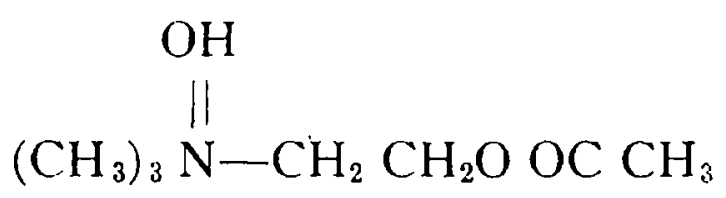

De plus, dans sa structure, nous remarquons plusieurs détails communs à un grand nombre de relaxants musculaires. La molécule contient deux atomes de nitrogène quaternaire, ou groupes onium, placés à distance l'un de l'autre. Ces groupes onium sont suffisamment basiques pour former un sel avec un acide fort. Ce sel réagit ensuite avec l'eau pour donner des solutions acides.

Cependant la succinylcholine differe de bien d'autres relaxants musculaires par cette propriété qu'elle a de s'hydroliser. Ceci serait da à la présence de deux groupes esther dans sa chaîne et expliquerait sa brièveté d'action brièveté relative.

L'effet principal de la succinylcholine est l'inhibition ${ }^{7,8}$ de la transmission neuro-musculaire. Cette action diffère de celle du curarisant vrai. Ce dernier augmente le seuil d'excitabilité de la plaque myoneurale tout en abaissant le degré d'efficacité déplorisante de l'acétylcholine. Ceci explique pourquoi les antagonistes de la cholinestérase (prostigmine, nệostigmine) sont des antidotes aux curarisants vrais, et non à la succinylcholine.

L'administration de succinylcholine entraine un bloquage neuro-musculaire sèmblable à l'injection d'une haute dose d'acétylcholine. Cette action est due à son aptitude intrinsèque d'effet dépolarisant sur la plaqué myoneurale et sur les fibres musculaires environnantes. La cholinestérase ne la détruisant pas, l'effet de dépolarisation se prolonge. Les anticholinestérases n'ont par conséquent aucune action.

Les muscles sont paralysés dans l'ordre suivant: les muscles de l'œil, de la face et du cou d'abord, les muscles des membres ensuite, puis ceux de l'abdomen et des intercostaux et enfin le diaphragme.

La succinylcholine n'est pas détruite par la cholinestérase ${ }^{9.10}$ proprement dite. Bien plus, les anticholinestérases prolongent l'action de la succinylcholine et sont donc absolument contre-indiqfées, car elles détruisent aussi la pseudocholinestérase responsable elle-même de la destruction de la succinylcholine. Cette destruction est faite en deux temps: 
(a) Formation de succínylmonocholine et de choline. La première aurait une action plus prolongée que sa parente qui est la succinyldicholine.

(b) Formation de choline et d'acide succinique, constituants normaux du plasma.

\section{MÉTHODES EMPLOYÉES}

Dans le laboratoire de chirurgie expérimentale, 82 chiens ont étê utilisés pour prélèvements de sang au cours des circulations extra-corporéales. Tous ces chiens étaient des bâtards. Leur poids variait de cinquante à cent vingt livres; et l'âge, de deux à quinze ans. Un diachylon autour de la gueule constituait la seule prémédication. Aucune anesthésie n'a été faite, car ce sang devait être exempt de toute substance anesthésique ou analgésique. Aussi la seule drogue employée a été la succinylcholine. Une seringue à insuline a été utilisée pour l'injector. La ponction veineuse a été faite dans la veine tibiale antérieure après rasage dư poil et désinfection locale. Les doses de succinylcholine ont varié de 6 à $8 \mathrm{mg}$.; $6 \mathrm{mg}$. pour les chiens plus petits et $8 \mathrm{mg}$. pour les chiens plus gros. Le produit employé avait une concentration de 10 pour cent, c'est-à-dire $20 \mathrm{mg}$. au c.c. En moyenne 10 secondes après l'injection de la succinylcholine, les paupières du chien se ferment et apparait une trémulation des muscles du front et du cou, suivie de la chute du chien; puis les sphincters se relâchent et le chien devient absolument flasque vingt-cinq secondes en moyenne après le début de l'injection de la succinylcholine. L'intubation endo-trachéale est faite dans les trente à soixante secondes qui suivent l'injection et la ventilation contrôlée est pratiquée. Le diaphragme, dernier muscle à se paralyser, s'arrête 40 à 60 sscondes après l'injection. La durée d'action de ces doses de succinylcholine a été de 15 à 20 minutes, avec une moyenne de 16 minutes.

Les doses de succinylcholine ont été réduites à $6 \mathrm{mg}$. puis à $4 \mathrm{mg}$. toujours en utilisant une seringue à insuline. Mêmes constatations que plus haut et même durée d'action. D'autre part, plus l'animal est âgé, plus la durée d'action est prolongée.

Chez tous ces chiens qui n'avaient reçu aucune prémédication, les constations suivantes ont été enregistrées après injection de succinylcholine:

(a) Un ralentissement marqué des battements cardiaques allant jusqu'à 20 à la minựte. Normalement le cour du chien bat de 80 à 120 à la minute. Ce phénomène a cédé spontanément 2 à 3 minutes plus tard à la suite de la ventilation contrôlée.

(b) Une arythmie qui a cédé en même temps que la bradycardie.

(c) Une sécrétion salivaire excessivement abondante dans tous les cas.

La moitié des chiens ont été gardés en vie pour être utilisés à nouveau comme donneurs de sang. Par exemple un chien pesant $126 \mathrm{lb}$. a été saigné 4 fois. La première fois il a reçu $8 \mathrm{mg}$. de succinylcholine, c'est-à-dire $0.06 \mathrm{mg}$. environ par livre de poids. Les constatations énumérées plus haut ont été observées. A la deuxième et à la troisième intervention cet animal a reçu $6 \mathrm{mg}$. et $4 \mathrm{mg}$. de succinylcholine, mêmes constatations. A la quatrième et dernière saignée, $3 \mathrm{mg}$. de succinylcholine seulement ont été injectés, c'est-à-dire $0.024 \mathrm{mg}$. par livre de poids. Les constatations et les résultats obtenus furent exactement les mêmes. 
Ces chiens ont été saignés tous les mois environ, laps de temps nécessaire pour refaire une formule sanguine normale.

Dans un deuxième groupe d'expériences, l'étude de doses répétées de succinylcholine a été faite. Quatre chiens font l'objet de cette étude.

Avant de commencer l'expérience proprement dite, avec la succinylcholine l'installation suivante a été faite sur chaque chien:

(a) Ponction de la veine tibiale antérieure à l'aide d'une aiguille reliée à un "stopcock" (appareil qui permet d'injecter mais n'autorise pas le retour sanguin).

(b) Anesthésie du chien au penthotal et intubation endotrachéale mais l'animal doit respirer spontanément. (En moyenne $150 \mathrm{mg}$. d'une solution de penthotal à $2 \frac{1}{2}$ pour cent a été suffisante dans tous les cas.)

(c) Désinfection de la région fémorale au zéphiran. Dissection de l'artère fémorale et mise en place d'un tube de polyéthylène dans cette artère. Ce tube de polyéthylène est relié à un appareil à $\mathrm{Hg}$ et à un "Transducer" pour enregistrer la pression d'une façon continue.

(d) Installation de l'électrocardiographe et de l'électroencéphalographe. Pour avoir un tracé électrocardiographique identique à celui de l'humain, les électrodes sont inverséés au niveau des pattes antérieures.

(e) Au cours de l'expérience, le chien sera relié au moyen du tube endotrachéal à un respirateur automatique fonctionnant sous air comprimé.

Après tous ces préparatifs, la succinylcholine sera injectée à l'aide d'une seringue à insuline. La période d'apnée sera le laps de temps qui s'écoule entre l'heure de l'arrêt de la respiration après l'injection de la succinylcholine et l'heure où le chien se ventilera spontanément sans aucune cyanose: la langue est le témoin le plus fidéle.

\section{Chien no 71}

Ce chien de $34 \mathrm{lb}$. a reçu comme prémédication: morphine $1 / 6$ de gr. et atropine $1 / 150$ de gr. Une première dose de $3 \mathrm{mg}$. de succinylcholine est injectée; 10 secondes plus tard la respiration s'arrête et ne reprend de façon satisfaisante qu'après 12 minutes. Lne deuxiême injection de $3 \mathrm{mg}$. entraîne une période d'apnée de 13 minutes et une troisième injection de $3 \mathrm{mg}$., une apnée de 24 minutes. Pendant tout ce temps, le chien bave abondamment malgré la prémédication.

\section{Chien no 28}

Ce chien de $40 \mathrm{lb}$. a reçu, une heure avant l'expérience, morphine $1 / 6 \mathrm{gr}$. et atropine 1/150 gr. Puís $4 \mathrm{mg}$. de succinycholine sont injectés rapidement par voie i.v. La période d'apnée est de 20 minutes. Une deuxième dose de $4 \mathrm{mg}$. est injectée et 30 minutes plus tard, la respiration spontanée n'est pas encore apparue. A ce moment $5 \mathrm{mg}$. de prostigmine sont injectés. L'electrocardiogramme montre des altérations cardiaques: ralentissement du cœur avec arythmie et troubles de la conduction. La T.A. cependant demeure normale. Si le respirateur est débranché du tube endotrachéal, la T.A. monte graduellement et le cœur devient de plus en plus irrégulier: effets dûs à l'anoxie. La douleur (par exemple, la torsion des orteils) provoque également une élévation de la T.A. et une accélération du 
pouls. Donc la succinylcholine n'enlève pas la douleur. La salivation est également abondante malgré la prémédication. Une troisième dose de $8 \mathrm{mg}$. est injectée et il faut attendre 60 minutes pour obtenir une respiration spontanée, qui ici cependant, ne se fait qu'aux dépens du diaphragme. Le chien est extubé. La T.A. monte graduellement, le pouls devient de plus en plus irrégulier et l'animal meurt dans les minutes qui suivent.

Chien no 19

Ce chien de $26 \mathrm{lb}$. ne reçoit aucune prémédication. Une première dose de succinylcholine de $3 \mathrm{mg}$. est administrée, elle entraîne une période d'apnée de 15 minutes. L'attention est d'abord portée sur la nature des preimers mouvements respiratoires. Ceux-ci débutent au diaphragme. L'hypersalivation est toujours enregistrée. Une deuxième injection de $3 \mathrm{mg}$. entraîne une apnée de 20 minutes. Cette apnée dure 35 minutes après l'administration de $6 \mathrm{mg}$. de succinylcholine, 60 minutes après l'injection de $12 \mathrm{mg}$. La respiration ne se fait qu'aux dépens du diaphragme et la cyanose de la langue apparait dès que le respirateur est débranché. Le chien est quand même désintubé mais il se cyanose de plus en plus et meurt au bout de quelques minutes noyé dans ses sécrétions.

\section{Chien no 60}

Ce chien pesant $28 \frac{1}{2} \mathrm{lb}$. ne reçoit aucune prémédication; $4 \mathrm{mg}$. de succinylcholine sont d'abord injectés. La T.A. monte brusquement de $140 \mathrm{~mm}$. de $\mathrm{Hg}$ à $160 \mathrm{~mm}$. de $\mathrm{Hg}$ pour s'abaisser en 30 secondes et redevenir normale en 60 secondes. Le tracé E.C.G. n'est aucunement modifié mais la' période d'apnée dure 15 minutes. Puis $8 \mathrm{mg}$. de succinylcholine sont injectés. La T.A. monte brusquement jusqu'à $180 \mathrm{~mm}$. de $\mathrm{Hg}$. Le cœur reste régulier, 20 minutes après cette troisième dose le chien ne respire pas encore. Après une quatrième dose de $30 \mathrm{mg}$., même poussée de la T.A. Celle-ci fait un bond à $200 \mathrm{~mm}$. de $\mathrm{Hg}$ pour redescendre ensuite graduellement. Le cœur fait quelques petites pauses. Les pupilles sont extrêmement dilatées; il n'existe aucun réflexe. Il faudra attendre 3 heures pour obtenir une respiration spontanée satisfaisante et désintuber le chien.

\section{Discussion}

S'il est admis que tout le règne animal* réagit de façon identique à l'administration d'une drogue (l'homme est un animal) il est possible de tirer les conclusions suivantes et d'en discuter:

(a) Les doses de succinylcholine efficaces sont peu élevées, dans l'ordre de 0.05 mg. à $0.07 \mathrm{mg}$. par livre de poids dans le laboratoire; alors que, actuellement dans la pratique courante de l'anesthésie, la succinylcholine se donne à la dose de $0.3 \mathrm{mg}$. à $0.5 \mathrm{mg}$. et même davantage par livre de poids chez un même individu au cours d'une même anesthésie.

Couramment, dans la littérature, il est dit que la dose de succinylcholine efficace varie de 40 à $80 \mathrm{mg}$. chez l'être humain adulte suivant son poids. Cela

*Pour démontrer cette assertion il faudrait faire des études chimiques plus poussées, en particulier doser la cholestérinase et la pseudo-cholestérinase dans le sang du chien et comparer les dosages à ceux rencontrés chez l'homme. 
veut dire que si la dose moyenne est de $50 \mathrm{mg}$. un individu de $160 \mathrm{lb}$. recevra $0.3 \mathrm{mg}$. par livre de poids; cette dose est beaucoup supérieure à la dose efficace trouvée au laboratoire.

A l'Hôtel-Dieu St-Vallier de Chicoutimi, les doses de succinylcholine employées pour intuber les malades ont été réduites sensiblement. Actuellement avec 20 mg. et même $15 \mathrm{mg}$., il est possible d'intuber tout adulte à condition d'y mettre un peu de dextérité et de rapidité.

(b) La durée d'action pour une première dose de succinylcholine est de 12 à 20 minutes. Plus cette dose est répétée plus la période d'apnée se prolonge. Il y a donc un effet cumulatif. Cela nous amène à douter de l'absence de danger du goutte à goutte intra-veineux ${ }^{8}$ et surtout de l'absence de danger de l'administration de la succinylcholine par voie intra-musculaire. ${ }^{11}$ En effet l'administration intra-musculaire peut échapper à tout contrôle d'absorption et partant de destruction. Si pour une raison quelconque l'absorption est retardée, le malade retourne à la salle de réveil, voire même à sa chambre d'hôpital acçusant une ventilation pulmonaire nettement insuffisante. Ce qui engendre la cyanose, l'atélectasie pulmonaire voire la broncho-pneumonie chez certains opérés elı particulier âgés. L'effet primordial de la succinylcholine, en effet, est de paralyser les muscles respiratoires. D'autres facteurs évidemment entrent en cause pour expliquer la dépression respiratoire au cours de la période postopératoire, tels: la prémédication, l'anesthésie, le choc opératoire-mais la succinylcholine a un effet prépondérant.

(c) La succinylcholine engendre l'hypersalivation, autre obstacle très important à la ventilation pulmonaire pour un individu qui arrive de la salle d'opération.

(d) L'administration de prostigmine ${ }^{1}$ ne neutralise en rien l'effet de la succinylcholine. Au contraire, elle semble en prolonger l'action.

(e) L'administration de succinylcholine à faible dose n'a peu ou pas d'effet sur la tension artérielle ni sur le rythme cardiaque. A haute dose cependant la succinylcholine augmente la T.A. et entraine des modifications de la conduction intra-cardiaque.

A la lumière des quelques données résumées au début, à la lumière aussi des constatations et des résultats enregistrés au laboratoire, il est très probable que les doses de succinylcholine employées à la salle d'opération sont trop élevées. Z'anesthésie se fait habituellement sur des malades où les facteurs suivants peuvent influencer la durée d'action de la succinylcholine ${ }^{7}:^{5}$

(a) Il peut y avoir un taux insuffisant de pseudocholinestérase dans le sang. Normalement le taux de cette enzyme est présentidans les proportions de 65 à 110 unités au c.c. Elle rest détruite par la prostigmine, tout comme la cholinestérase. Le taux de pseudocholinestérase est beaucoup plus élevé chez l'enfant que chez l'adulte et est très bas chez le vieillard. D'autre part un taux abaissé de pseudocholinestérase est retrouvé dans les cạs suivants: après radiations thérapeutiques; après contaminations par substances phosphorées dues aux insecticides; dans les états d'hyperpyrexie; au cours des insuffisances cardiaques; au cours des états urémiques; dans-les maladies du foie; au cours des maladies de dénutrition; dans les anémies sévères; dans l'hyperthyroidisme; chez tout grand malade. 
(b) Il peut y avoir destruction incomplète de la succinylcholine. Il a été dit plus haut que la succinylmonocholine, produit intermédiaire, avait une action plus prolongée que sa parente la succinyldicholine. De plus la choline elle-même quand elle est présente en excès, aurait une action de bloquage sur la plaque myoneurale; excès qui pourrait être dû à l'administration de trop fortes doses.

(c) Il peut y avoir acapnie ou hypercapnie dues à l'hyper ou l'hypoventilation assistée ou contrôlée au cours de l'apnée créée par la succinylcholine, empêchant ainsi l'action normale du centre bulbaire respiratoire.

(d) Il peut y avoir chambardement du réflexe de Hering-Breuer dû à la distension en plus ou en moins des alvéoles pulmonaires au cours de la respiration contrôlée ou assistée.

(e) 11 peut y avoir hyper ou hypocalcémie. ${ }^{13}$

(f) Il peut y avoir association de drogues: barbituriques-opiacés qui dépriment le centre respiratoire.

(g) Il peut y avoir inactivation de la pseudocholinestérase par la sérotine. ${ }^{4}$

\section{RÉSUMÉ ET CONCLUSION}

Les doses de succinylcholine employées en anesthésie actuellement seraient, à notre avis, trop élevées et certaines complications pulmonaires post-opératoires seraient attribuables à cette drogue (atélectasie, broncho-pneumonie etc.).

Après avoir donné un brief résumé sur l'historique de la succinylcholine, sur sa constitution chimique sur son mode d'action ainsi que sur son sort dans l'organisme, les résultats obtenus dans le laboratoire sont exposés.

Dans une première série d'expérience, 82 chiens ont été utilisés comme donneur de sang au cours de circulations extra-corporéales. Chez ces 82 chiens, la dose efficace de succinylcholine administrée a varié de $0.024 \mathrm{mg}$. à $0.06 \mathrm{mg}$. par livre de poids. Dans une deuxième série d'expériences, 4 chiens ont fait l'objet d'une étude plus complète; les conclusions ont été les suivantes:

(a) La dose de succinylcholine efficace varie de 0.03 à $0.07 \mathrm{mg}$. par livre de poids dans le laboratoire.

(b) La succinylcholine administrée à doses répétées a un effet cumulatif.

(c) La succinylcholine engendre l'hypersalivation en dépit de la prémédication.

(d) La succinylcholine à hautes doses a un effet hypertensif malgré la prémédication et malgré l'anesthésie au penthotal.

(e) La prostigmine a un effet nocif en présence de la succinylcholine.

Dans le service d'anesthésie de l'Hôtel-Dieu St-Vallier de Chicoutimi, les doses de succinylcholine administrées aux malades ont été sensiblement réduites tout en conservant les mêmes résultat.

\section{RECONNAISSANCE}

Nous remercions de façon spéciale l'equipe de nos collaborateurs immédiats: mademoiselle Charlotte Lavoie, i.l., en charge du service interne chirurgical du service de chirurgie cardio-vasculaire et ses collaboratrices: mademoiselle Jeanne Viel, i.l., mademoiselle Jacqueline Gilbert, i.l., mademoiselle Solange Favreau, i.l., Monsieur Marcel Gagné, diplôme en technologie électronique et spécialisé dans le fonctionnement du cœur artificiel, monsieur Marc Buteau, assistant technicien et monsieur Cormier en charge du chenil. 


\section{BIBLIOGRAPHIE}

1. Mayrhofer, O. K. Prolonged Apnea Following Scoline. Anaesthesia 7: 250 (Oct., 1952).

2. Goodman, Louis S., \& Gilman, Alfred. The Pharmalogical Basis of Therapeutics. 2nd ed, New York: Macmillan (1955).

3. Bovet, D., ET AL.

Proprieta Farmacodinamiche di Alcuni Derivati Della Succinicolina Doti de Axione Curarica, Rendic. Ist. Super. San. 12: 106-138 (1949).

Recherches sur les poisons curarisants de synthèse. Arch. Internat. Pharmacodyn 88 : 7 (1951).

4. Phillips, A. P. Synthetic Curare Substitutes from Aliphatic Dicarboxilic Acid Aminoethyl esters. J. Am. Chem. Soc. 71: 3264 (1949).

5. Low, K., \& Tammelin, L. E. Nord. Med. 46: 1043 (1951). Acta Physiol. Scand. 23: 78 (1957).

6. Hampton, Jennings L.; Little, David M.; Rodabaagh, Galon S.; \& Chaffee, William R. Studies Concerning the Prolongation of Activity of Succinylcholine. Anesthesiology 16 (Jan., 1955).

7. LeE, J. Alfred. A Synopsis of Anaesthesia. 4th ed. pp. 395ff. (1959).

8. Hewer, Langton C., \& LeE, J. Alfred. Recent Advances in Anaesthesia and Analgesia. 8th ed. pp. 112ff. (1957).

9. Evans, F. T.; Gray, P. W. S.; Lehman, H.; \& Silk, E. Sensitivity to Succinylcholine in Relation to Serum-Cholinesterase. Lancet $i: 1229$ (1952).

10. KaIow, W. The Relation of Plasma Cholinesterase to Response to Clinical Doses of Succinylcholine. Canad. Anaesth. Soc. J. 3: 32 (1596).

11. Beldavs, J. Intramusqular Succinylcholine for Endotracheal Intubation in Infants and Children. Canad. Anaфsth. Soc. J. 6: 141 (1959).

12. Irwin, Richard L., Welís, Jay B., \& Whitehead, Richard W. Effect of Calcium and the Duration of Apnea Induced by Succinylcholine. Anaesthesiology 17 (Nov.-Dec., 1956).

13. Jones, W. E. G. Serotonin and the Carcinoid Syndrome. Canad. Anaesth. Soc. J. 16: 130 (1959). 